\title{
EFECTOS DE LA SEQUÍA DEL AÑO 2017 SOBRE LA NUBOSIDAD EN LA PENÍNSULA IBÉRICA
}

\author{
Dominic ROYÉ \\ Universidade de Santiago de Compostela \\ dominic.roye@usc.es \\ Nieves LORENZO \\ Universidade de Vigo
}

\begin{abstract}
Resumen
El año 2017 fue un año climáticamente anómalo tanto en temperatura como en precipitación para la Península Ibérica. El patrón de precipitaciones se encuentra íntimamente relacionado con la nubosidad, y a su vez, las nubes juegan un papel fundamental en el sistema terrestre con fuertes efectos en los procesos atmosféricos. Las nubes son una pieza clave del estudio del clima y especialmente en la comprensión del cambio climático. Para conocer los efectos que tuvo la sequía en la nubosidad, en este estudio se hace uso de datos de observación satélite (MODIS). Además, con el objetivo de encontrar causas para las anomalías observadas se estimaron los tipos de circulación. Los resultados indican una clara correlación entre las anomalías de precipitación y las observadas en la fracción de nubosidad. La sequía de 2017 se caracterizó por un comportamiento de la circulación atmosférica similar al registrado en las tres sequias más severas sufridas en los últimos 11 años. La principal causa podemos encontrarla en una circulación anticiclónica y de tipo norte bastante superior a la media, e inversamente se registró una clara disminución de situaciones ciclónicas y de flujos procedentes del oeste.
\end{abstract}

Palabras clave: sequía, nubosidad, península ibérica, precipitación, tipos de circulación

\section{Resumo}

O ano de 2017 foi um ano climaticamente atípico tanto em temperatura como em precipitação na Península Ibérica. O padrão de precipitação encontra-se intimamente relacionado com a nebulosidade, e por sua vez, as nuvens apresentam um papel fundamental no sistema terrestre com fortes efeitos nos processos atmosféricos. As nuvens são uma peça chave para o estudo do clima e especialmente para a compreensão das alterações climáticas. Para conhecer os efeitos da seca na nebulosidade, neste estudo utilizam-se dados de satélite (MODIS). Para além disso, com o objetivo de encontrar causas para as anomalias observadas, estimaram-se os tipos de circulação. Os resultados indicaram uma evidente correlação entre as anomalias de precipitação e as observadas na fração de nebulosidade. A seca de 2017 caracterizou-se por um comportamento da circulação atmosférica similar ao registado nas três últimas secas mais severas sofridas nos últimos 11 anos. A principal causa apontada está na circulação anticiclónica e do tipo norte bastante superior à media, e inversamente, registou-se uma clara diminuição de situações ciclónicas e de fluxos procedentes do oeste.

Palavras-chave: seca, nebulosidade, Península lbérica, precipitação, tipos de circulação

\section{Introduccion}

El año 2017 fue un año climáticamente anómalo tanto en temperatura como en precipitación para la Península lbérica $(\mathrm{Pl})$. Según los datos registrados por la agencia estatal de meteorología 
(AEMET), 2017 tuvo la temperatura media más alta desde 1965 y debemos retrotraernos a 2005 para registrar valores menores de precipitación media $(474 \mathrm{~mm})$. Estos episodios cada vez parecen ser más recurrentes en los últimos años. Desde la ola de calor de 2003 se han registrado siete años con temperaturas anómalamente elevadas y de esos siete años 5 se concentran en los últimos diez años. La anterior sequía de magnitud similar en la PI tuvo lugar en 2005 con 468 mm de precipitación media. Los años 2015, 2011, 2012 y 2007 también registraron precipitaciones por debajo de los 600 $\mathrm{mm}$. Estos datos corroboran las proyecciones de cambio climático para el siglo XXI, que pronostican una $\mathrm{PI}$ con un clima más extremo dominado por olas de calor, periodos de sequía y precipitaciones torrenciales. Estos periodos de sequía tienen importantes secuelas en diferentes ámbitos socioeconómicos afectando a campos tan desiguales como el de la agricultura, el turismo o el de la producción eléctrica. Durante 2017 la producción hidráulica se situó en 18.364GWh, un 49\% inferior a la de 2016 y la más baja desde el año 2005. De esta forma, la hidráulica contribuyó tan solo un 7,0\% al total de la producción nacional, muy inferior a la contribución del 13,7\% registrada en 2016.

El patrón de precipitaciones se encuentra íntimamente relacionado con la nubosidad. Las nubes juegan un papel fundamental en el sistema terrestre con fuertes efectos en los procesos atmosféricos, son una pieza clave en el estudio del clima y especialmente en la comprensión del cambio climático. Las nubes, así como los gases de efecto invernadero, pueden provocar un calentamiento del planeta al atrapar el calor que se encuentra debajo de ellas, pero también pueden tener un papel de enfriamiento al reflejar los rayos que provienen del sol. Además, en el proceso de formación de nubes, la temperatura del aire también se ve afectada. En particular, las nubes son un elemento clave en el balance de radiación, causando variabilidad en la radiación infrarroja solar y atmosférica debido a su alto grado de albedo. Otro papel clave importante existe en su participación en el balance hídrico global. En consecuencia, existen múltiples interacciones complejas con la superficie terrestre, que pueden influir en los patrones espacio-temporales de temperatura y precipitación. El calentamiento del verano observado en Europa desde la década de 1980 acompañado por un aumento en la ocurrencia de olas de calor y, en consecuencia, déficits de agua, puede explicarse parcialmente por cambios en la nubosidad del verano (Tang et al., 2012). Siguiendo esta idea, este trabajo analiza cómo la nubosidad podría estar relacionada con la sequía, o bien cómo se refleja la sequía en la nubosidad, que ha afectado a la PI durante 2017. Para llevar a cabo este estudio se utilizó la cobertura de nubes diaria de los satélites MOD09GA y MYD09GA (versión 6) para calcular la fracción de nubes promedio en 2017 y para los diferentes meses del mismo año, que se compara con el promedio a largo plazo entre 2001 y 2017. Analizamos estas anomalías en relación con los cambios en la precipitación, la temperatura y los cambios en la circulación atmosférica durante 2017, para comprender mejor las relaciones entre la nubosidad, la precipitación y el cambio climático.

El objetivo de esta investigación es mostrar las principales características climáticas ocurridas durante la sequía sufrida por la PI en 2017 atendiendo a la circulación sinóptica y a los principales modos de circulación que afectan a la PI. El artículo se organiza de la siguiente manera: la Sección 2 describe el área de estudio y la base de datos de nube y datos de patrones sinópticos y modos de circulación utilizados en este estudio. En la sección 3, se muestran los resultados de la distribución 
espacial y temporal de la cobertura de nubosidad0 para el año 2017 en relación a la media del periodo 2001-2017 y se analizan las anomalías de circulación registradas en este periodo de sequía. Finalmente, en la sección 4 se resumen las principales conclusiones.

\section{2. Área de estudio}

La PI se encuentra al suroeste de Europa influenciada por climas tropicales y templados además de con un relieve complejo. Debido a esto y a la influencia del océano, presenta una marcada variabilidad climática con un gradiente de precipitación significativa norte-sur. La variabilidad climática de la PI está modulada principalmente por la Oscilación del Atlántico Norte (NAO), pero otros patrones como el patrón del Atlántico Este (EA) o el patrón del Atlántico Este/Rusia Occidental (EA/WR) también tienen una influencia notable en la variabilidad climática de esta región. (RodríguezPuebla et al., 1998,2001; Esteban-Parra et al., 1998; Muñoz-Díaz y Rodrigo, 2004; Aupí, 2005).

\section{Datos y métodos}

\subsection{Datos de teledetección}

Existen muchas técnicas disponibles para la detección de nubes, aunque solo la detección remota con instrumentos satelitales tiene la capacidad de recopilar y monitorizar continuamente la cobertura de nubes a gran escala y de manera eficiente. Los datos de la nubosidad en este estudio provienen del instrumento MODIS, un sensor a bordo de los satélites TERRA y AQUA, que producen varios productos de nube de alta resolución diarios (Baum y Platnick, 2006; Frey et al., 2008).Estos satélites tienen una órbita polar alrededor de la Tierra sincronizada con el Sol. Esto significa que recorre una trayectoria que pasa por ambos polos, caracterizada por aparecer cada día sobre un punto dado del ecuador siempre a la misma hora local. TERRA va de norte a sur pasando por el ecuador a las 10:30 A.M, mientras que AQUA orbita de sur a norte en el nodo ascendente y cruza por el ecuador a las 1:30 P.M.

En este estudio se utiliza la máscara de nubosidad de "State-1 km: Reflectance Data State", derivada de la máscara MOD35, en el producto atmosférico reticulado (colección 6) de la reflectancia global de superficie diaria L2G con una resolución de $500 \mathrm{~m}$ y $1 \mathrm{~km}$. (MOD/MYD/ 09GA) (Baum et al., 2012; Vermote \& Wolfe, 2015). El esquema de detección de nubosidad en MODIS intenta conservar los cielos despejados (Frey et al., 2008) y el algoritmo de máscara identifica varios dominios conceptuales según el tipo de superficie y la iluminación solar. Las mejoras de las colecciones 5 y 6 son discutidas por Frey et al. (2008) y Toller et al. (2013).

La capa "State-1 km: Reflectance Data State" incluye información sobre las condiciones de las nubes según la siguiente clasificación: cielo despejado (0), nublado (1), mixto (2) y datos faltantes (3). Para obtener valores entre 0 y 1 , el tipo mixto (2) se convierte a 0.5 y los datos faltantes se ocultan 
(Kotarba, 2010). La resolución de $1 \mathrm{~km}$ tiene un nivel de detalle suficiente para el objetivo de este estudio sobre la PI. El análisis del estudio cubre las series temporales diarias disponibles de MOD09GA y MYD09GA entre 2001 y 2017.

\subsection{Tipos de circulación}

La clasificación de los patrones atmosféricos se ha convertido en un tema importante en los estudios climáticos en los últimos años, ya que dichos patrones o tipos de circulación permiten integrar diferentes parámetros meteorológicos, como la lluvia, la dirección del viento, la intensidad y la temperatura en un solo índice. De esta forma a cada día se le asigna un tipo de circulación, haciendo posible estudiar la variabilidad meteorológica asociada y sus consecuencias de una manera sencilla (Huth et al., 2008; Philipp, 2009; Philipp et al., 2010; Ramos et al., 2015; Huth et al., 2016). En este estudio, se utiliza una clasificación que toma en cuenta los datos de SLP de NCEP-DOE Reanalysis 2. Estos datos se tomaron de una versión mejorada del modelo NCEP Reanalysis I que corrigió errores y actualizó las parametrizaciones de los procesos físicos. El procedimiento adoptado se desarrolló en Trigo \& Da Camara (2000), que, a su vez, se adaptó del desarrollado por Jenkinson \& Collison (1977) y Jones et al. (1993). Calculamos la dirección y la vorticidad de los flujos geostróficos, realizando cálculos a diario para el período 2001-2017 para el cual tenemos datos de cobertura de nubes.

\subsection{Datos de teleconexiones}

En lo referente a las teleconexiones que afectan a la PI, las series temporales se tomaron del centro de predicción climática de la NOAA (National Oceanographic and Atmospheric Administration) de Estados Unidos. Estos índices son calculados mediante un análisis de componentes principales a $500 \mathrm{hPa}$, y están disponibles en la página web www.cpc.noaa.gov/data/teledoc/telecontents.shtml.

\section{Resultados}

\subsection{Patrones de la nubosidad}

En la Figura 1 se muestra la fracción media de nubosidad registrada en el año 2017 frente a la fracción media de nubosidad para el periodo 2001-2017

Como se puede ver durante el año 2017 la nubosidad en toda la PI fue menor que la media de los últimos 17 años. Si analizamos la distribución de la fracción de nubosidad para este año comparándola con la referencia 2001-2017 (Figura 2), se puede observar que dicha función de densidad durante 2017 presenta dos particularidades a destacar. Por un lado, el máximo de menor fracción de nubosidad se encuentra significativamente desplazado hacia valores de fracción de nubosidad menores que los de la media, y además presenta una menor amplitud. Por otro lado, el 
segundo máximo de la función de densidad, aunque no muestra un desplazamiento significativo con respecto a los valores del periodo 2001-2017, sí presenta una menor amplitud. Esto corrobora lo observado en la Figura 1 dónde se aprecia una disminución generalizada de la fracción de nubosidad para todo el territorio peninsular. La diferencia se ve reflejada en este desplazamiento, mostrando un claro comportamiento bimodal distinguiendo las regiones de menor nubosidad localizadas al sur de la PI de las de mayor nubosidad localizadas al norte de la PI.

2001-2017

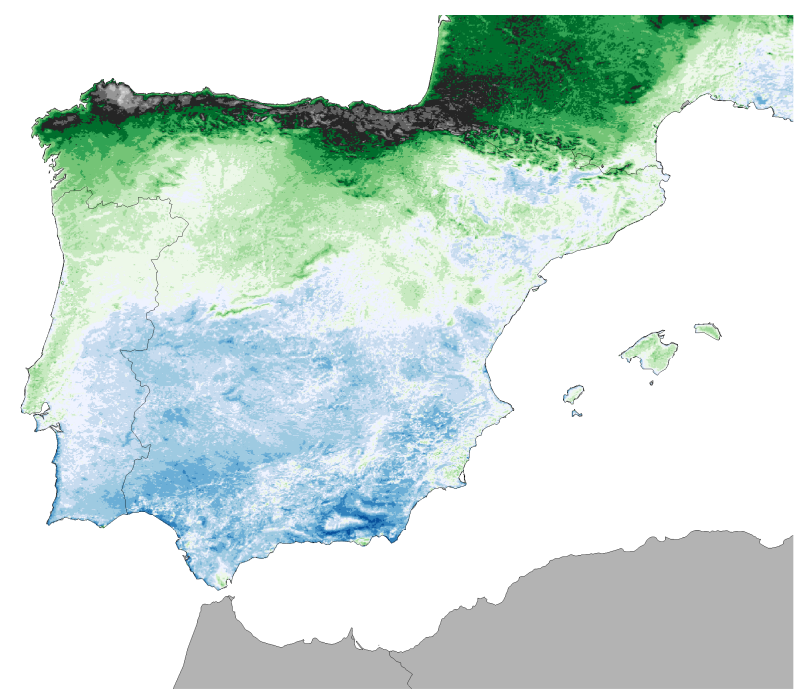

2017

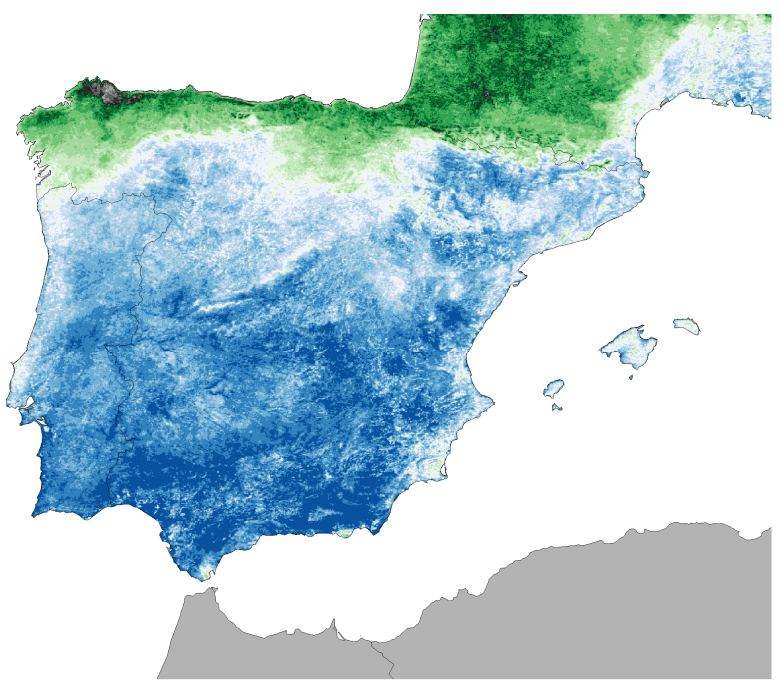

\begin{tabular}{|l|l|l|l|l|}
\hline$<0.31$ & $0.402-0.425$ & $0.514-0.551$ & $0.641-0.666$ & $0.735-0.76$ \\
\hline $0.317-0.349$ & $0.425-0.452$ & $0.551-0.587$ & $0.666-0.69$ & $>0.76$ \\
\hline $0.349-0.377$ & $0.452-0.482$ & $0.587-0.616$ & $0.69-0.713$ & \\
\hline $0.377-0.402$ & $0.482-0.514$ & $0.616-0.641$ & $0.713-0.735$ &
\end{tabular}

Figura 1: Fracción media de nubosidad para el año 2017 y para el periodo de referencia 2001-2017.

Cuando analizamos mensualmente la distribución de la fracción de nubosidad, observamos que los meses que muestran una mayor desviación de la nubosidad son los meses de primavera y otoño (Figura 3). Se trata de meses esenciales respecto al balance hídrico de la PI. Abril, septiembre, octubre y noviembre han sido los meses con una mayor anomalía en su nubosidad, con lo que consecuentemente asociado a esta menor nubosidad se encuentra asociada una menor precipitación. El mes de enero, aunque no de manera tan significativa, presenta una importante anomalía, desapareciendo en distribución casi por completo el segundo máximo asociado a la región con una mayor cobertura nubosa. Ello se refleja en la Figura 4, donde se ve la nubosidad media de enero para el periodo 2001-2017 en contraposición con la nubosidad media ocurrida durante el enero de 2017. Las regiones de la mitad norte y oeste de la PI, presentaron una disminución importante de 
nubosidad, no así la región este de la PI con valores similares a la media. Esta pérdida de nubosidad y consecuentemente de aporte hídrico en las regiones más húmedas de la PI en los meses considerados lluviosos, hizo que las reservas hídricas bajaran muy por debajo de la media y se llegara a niveles de sequía similares a los de la sequía registrada en 2005.

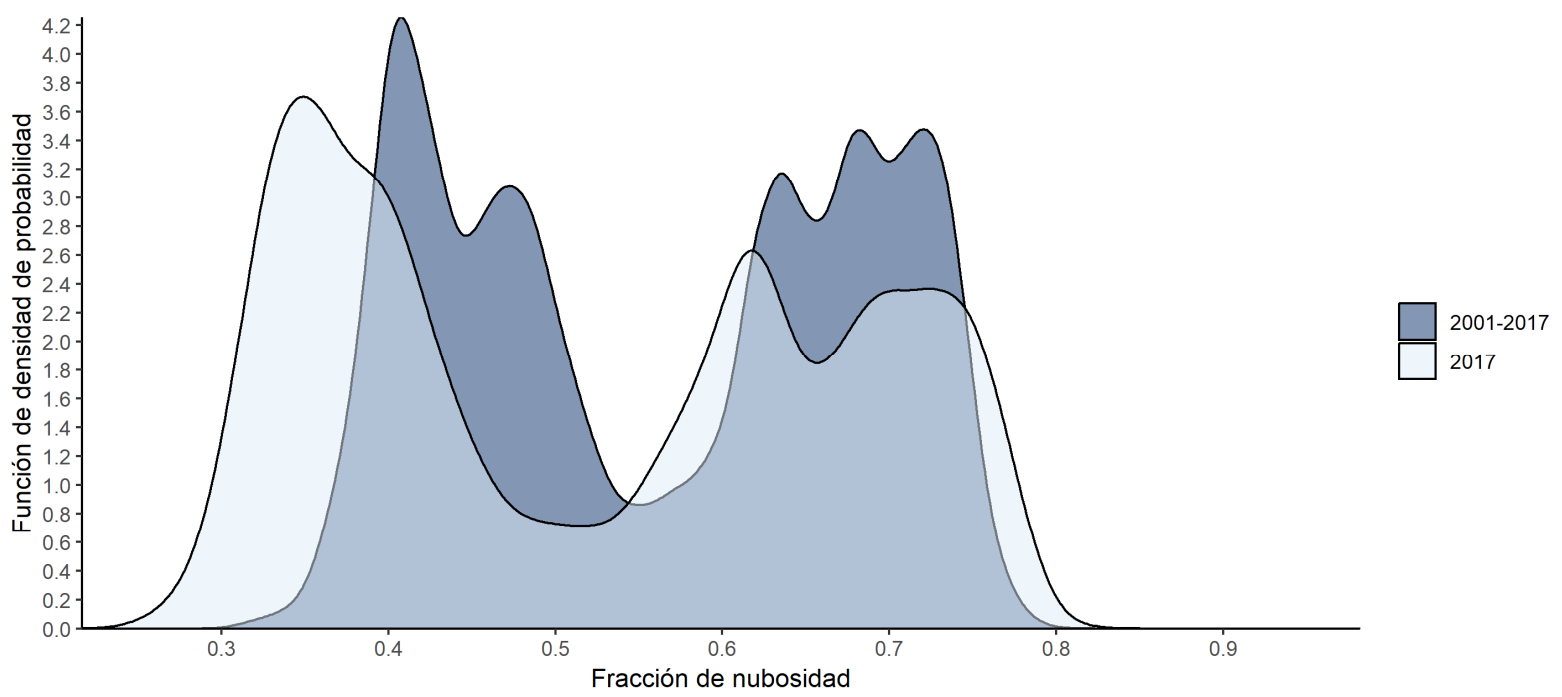

Figura 2: Función de densidad de probabilidad de la fracción promedio de nubosidad para el año 2017 y para el periodo 2001-2017.

Un comportamiento similar, aunque más acusado y extendido a toda la PI, tuvo lugar también durante los meses de abril y octubre, dos meses claves en el ciclo hidrológico de la PI. En la PI las lluvias tienen una distribución bimodal con un máximo en otoño y otro en primavera, aunque en la región más occidental el periodo de lluvias otoñal puede extenderse hasta el invierno.

La anomalía de precipitaciones registrada en los meses de primavera con un $23 \%$ por debajo de la media seguida de un otoño que registró una precipitación media sobre España de 84 mm, (59\% inferior al valor medio del trimestre) provocó una de las sequias más graves de la última década, dejando un 15\% de déficit respecto a la media histórica (1981-2010). El problema no es solo este año de sequía, sino que desde 2014 hemos ido encadenando años consecutivos con lluvias por debajo de la media histórica que se encuentra en el $648 \mathrm{l} / \mathrm{m}^{2}$.

Las consecuencias de esta sequía se tradujeron en anomalías importantes en la humedad del suelo (Figura 5) y en la productividad de la vegetación con graves consecuencias en el sector agrícola y en el de producción energética. La producción hidroeléctrica en España, como mencionamos en la introducción, es muy variable con valores, según datos de Red Eléctrica de España, que pueden llegar al $25 \%$ de la energía eléctrica total o quedarse por debajo del $8 \%$, como ocurrió en las sequias más severas de los últimos 30 años (1992, 2005, 2012). Durante la sequía del 2017 la producción se ha quedado en un $7 \%$, y es una de la más baja registrada hasta el momento (Figura 6). 

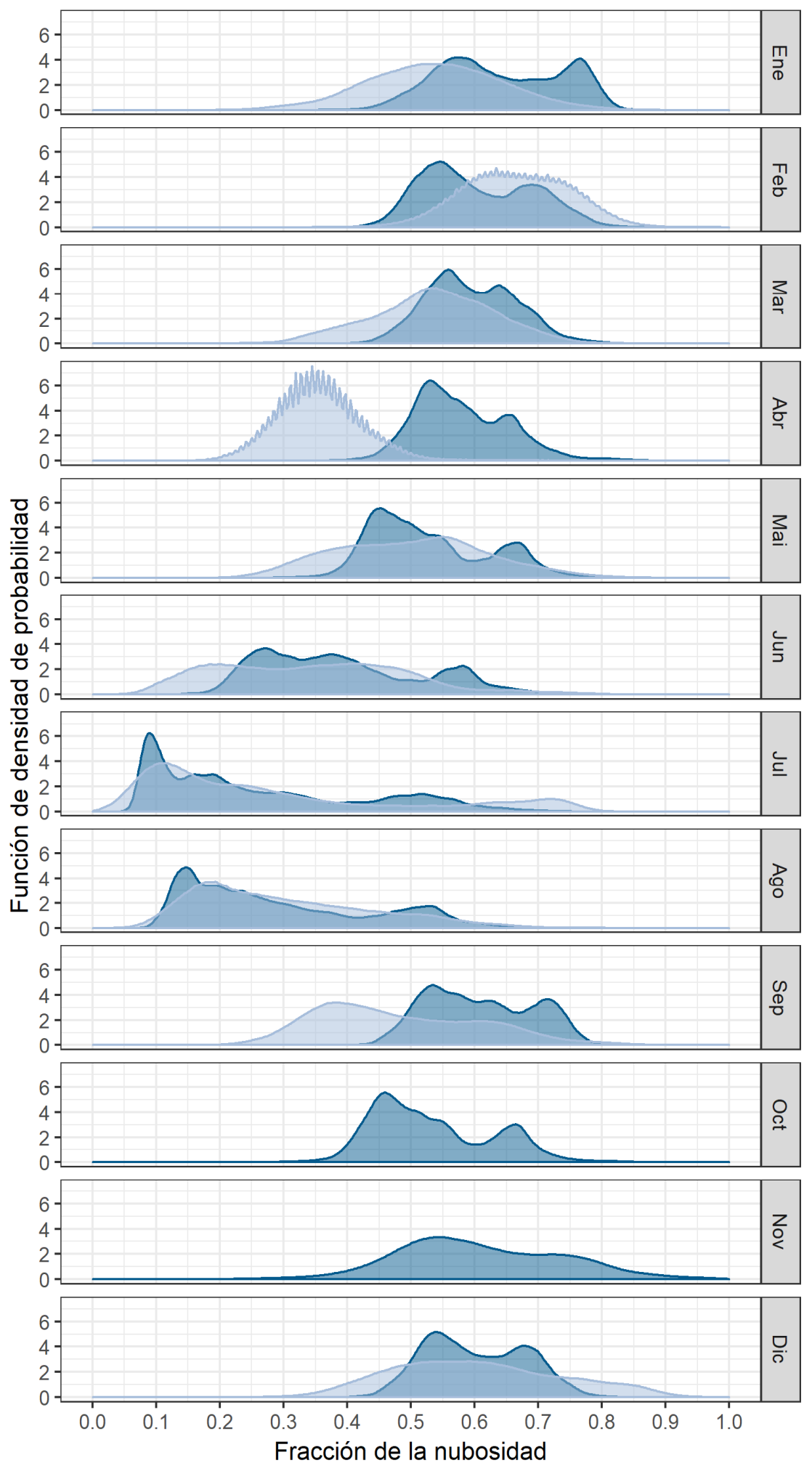

Figura 3: Función de densidad de probabilidad de la fracción promedio de nubosidad por mes para el año 2017 y para el periodo de referencia 2001-2017. 


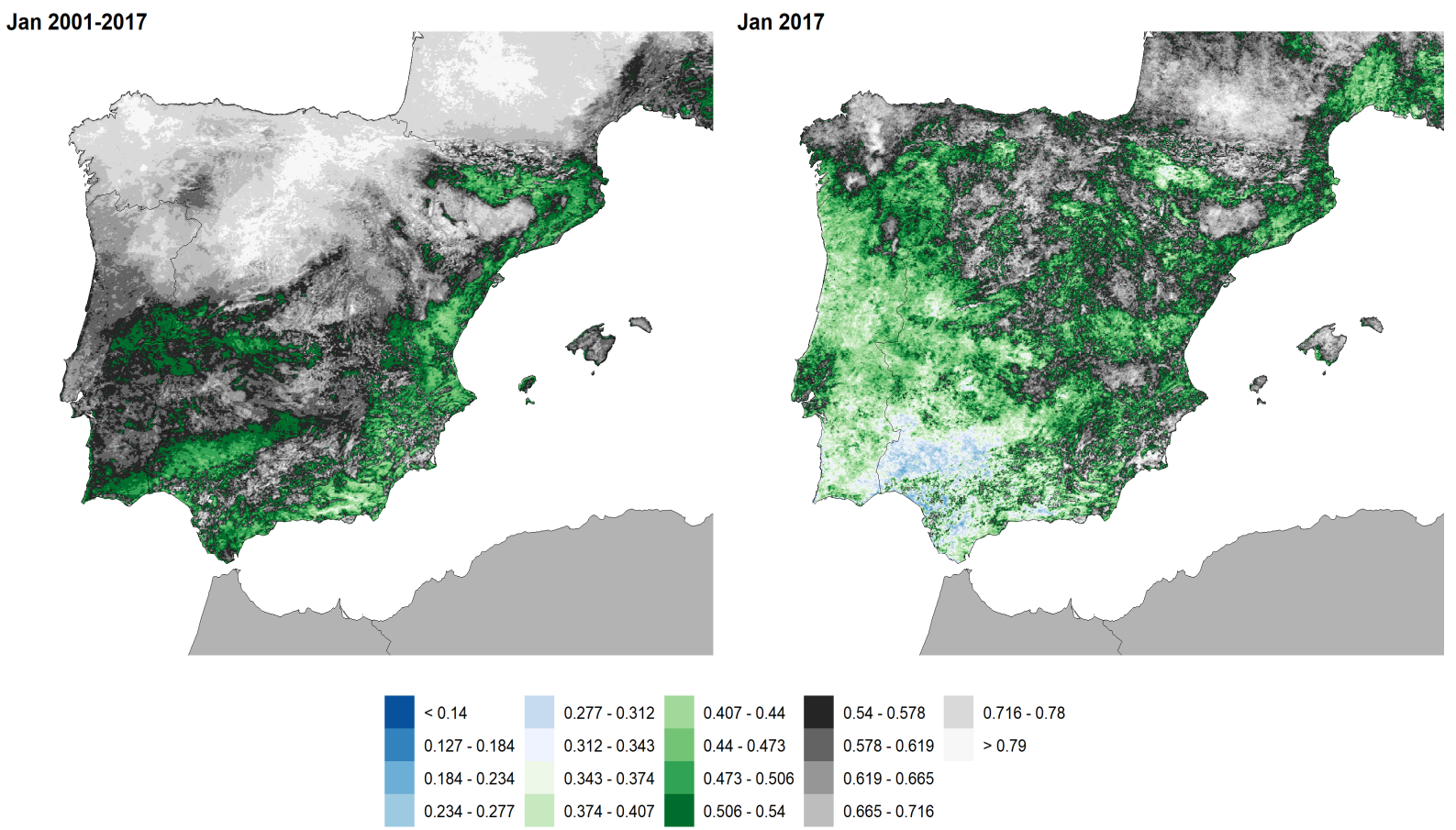

Figura 4: Fracción media de nubosidad (2001-2017) y 2017 para el mes de enero.
Abril
Mayo

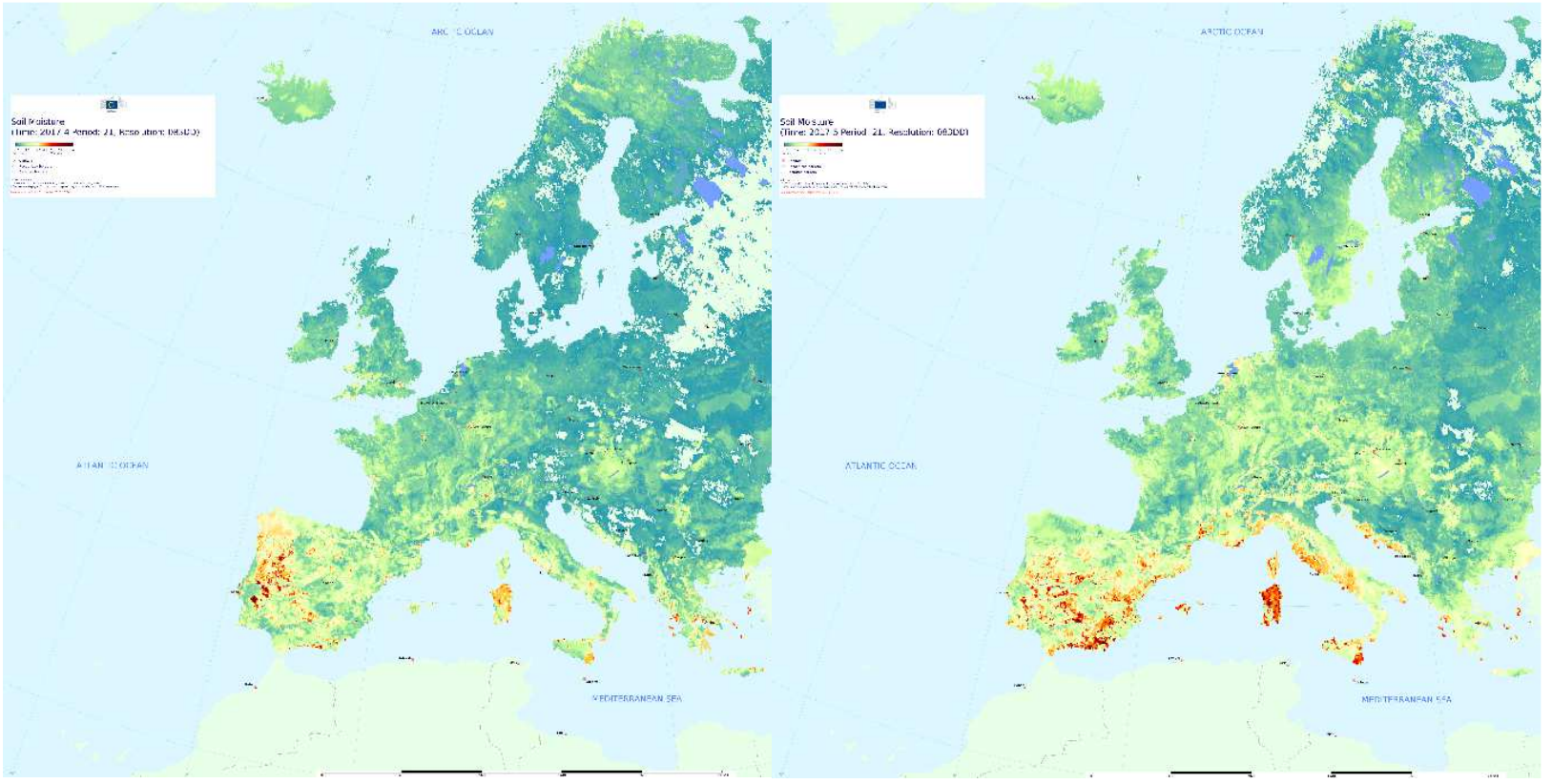

Figura 5. Anomalía de la humedad en el suelo en Europa de los meses abril y mayo de 2017.

Fuente: http://edo.jrc.ec.europa.eu 


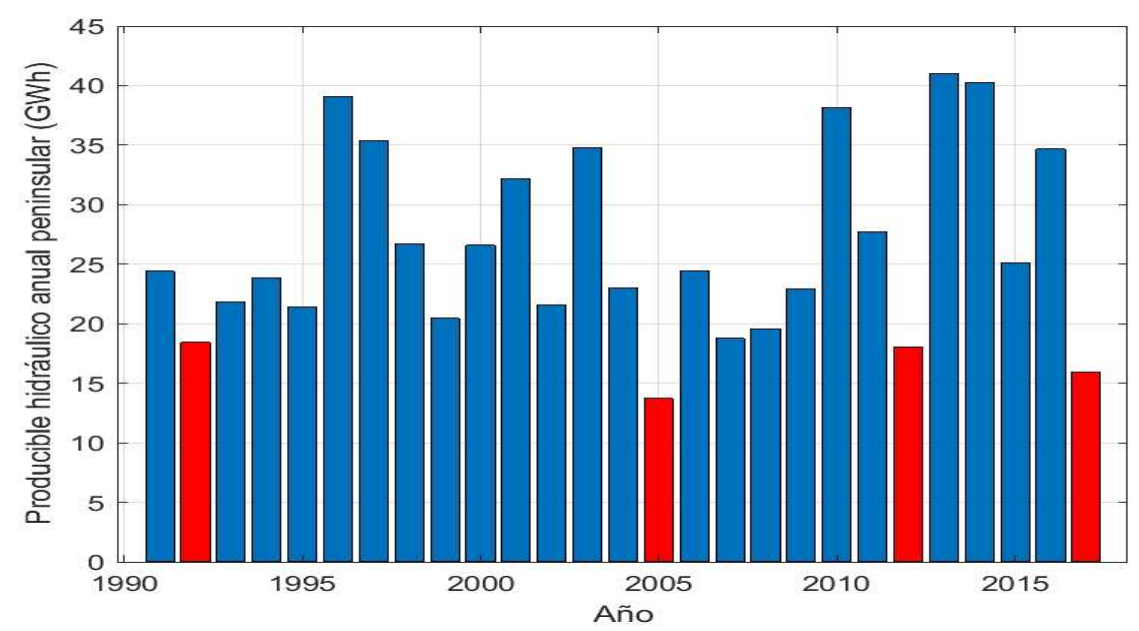

Figura 6. Producción de Hidroelectricidad anual. Fuente de datos: Red Eléctrica de España.

\subsection{Patrones globales y mecanismos físicos}

Un análisis de los patrones de circulación sinópticos sobre la PI nos muestra que durante el 2017 hubo una circulación anticiclónica y de tipo norte bastante superior a la media e inversamente se registró una clara disminución de situaciones ciclónicas y de flujos procedentes del oeste (Figura 7). Recientemente, Royé et al. (2018) hicieron el primer estudio relacionando los tipos de circulación con sus efectos en la nubosidad de la PI. En dicho estudio se observa que la mayor nubosidad registrada en los meses de invierno se debe al predominio de situaciones sinópticas zonales (W, SW, ASW) con predominio del flujo marítimo desde el océano Atlántico acompañado de sistemas frontales. Por el contrario la menor nubosidad se encuentra asociada a la prevalencia de situaciones estables (A, ANE, E, SE, etc.) y masas de aire con menor humedad. Los flujos del oeste son los que traen mayores precipitaciones a la PI (Lorenzo et al., 2008).

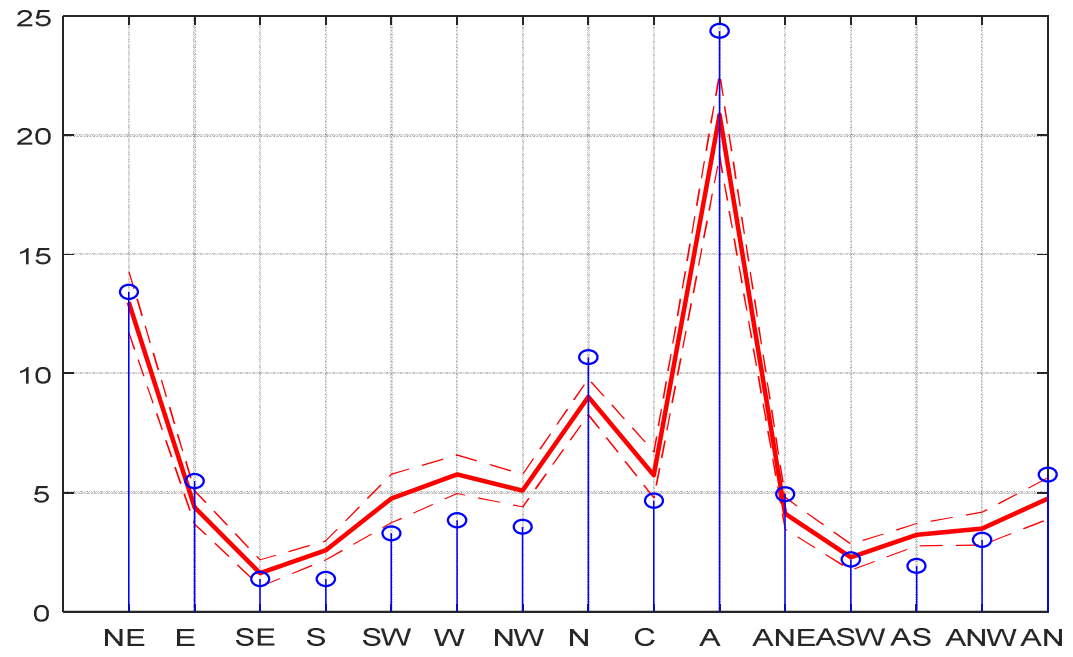

Figura 7: Anomalías de los tipos de circulación en 2017 en la PI con referencia al período 2001-2017. La línea roja continua es el porcentaje de frecuencia para el periodo 2001-2017, las líneas punteadas es la desviación estándar para el periodo 20012017. Los círculos azules se corresponden con el porcentaje de frecuencia del 2017. 
La razón de esta anomalía en los patrones o tipos de tiempo locales se encuentra íntimamente relacionado con los patrones de circulación globales. El patrón de teleconexión del sector euroatlántico que mayor influencia muestra sobre la PI es la Oscilación del Atlántico Norte (NAO) que tiene una influencia significativa y conocida desde hace tiempo en el régimen de precipitaciones de la PI (Trigo et al. 2004). Dicha oscilación modula el flujo atmosférico del oeste de tal forma que cuando presenta valores positivos las precipitaciones en la PI presenta valores por debajo de la media y viceversa con valores negativos los valores de precipitación son superiores a la media. Sin embargo, otros patrones como el de Atlántico este (EA), el del Atlántico Este/Rusia occidental (EA/WR), el escandinavo (SCAN) o el polar (POL) también pueden jugar un papel importante en la variabilidad de la precipitación de la PI (deCastro et al., 2006; Trigo et al. 2008). Durante el año 2017 tanto NAO como EA y EA/WR se mostraron mayoritariamente positivas, mientras que el índice SCA y POL fueron negativos. La conjunción de las fases de NAO, EA/WR y SCA favorece un periodo menos adecuado para la ocurrencia de precipitaciones, ya que NAO y EA/WR presentan una correlación negativa y SCA una correlación positiva con las precipitaciones de la PI (deCastro et al., 2006; Casanueva et al., 2014). Esta conjunción se dio también en las otras dos sequias más severas sufridas en los últimos 11 años la del 2005 y la del 2011-2012. Kingston et al. (2013) encontraron que una combinación de los patrones de circulación NAO y EA/WR son las teleconexiones más importantes en épocas de sequías para Europa.

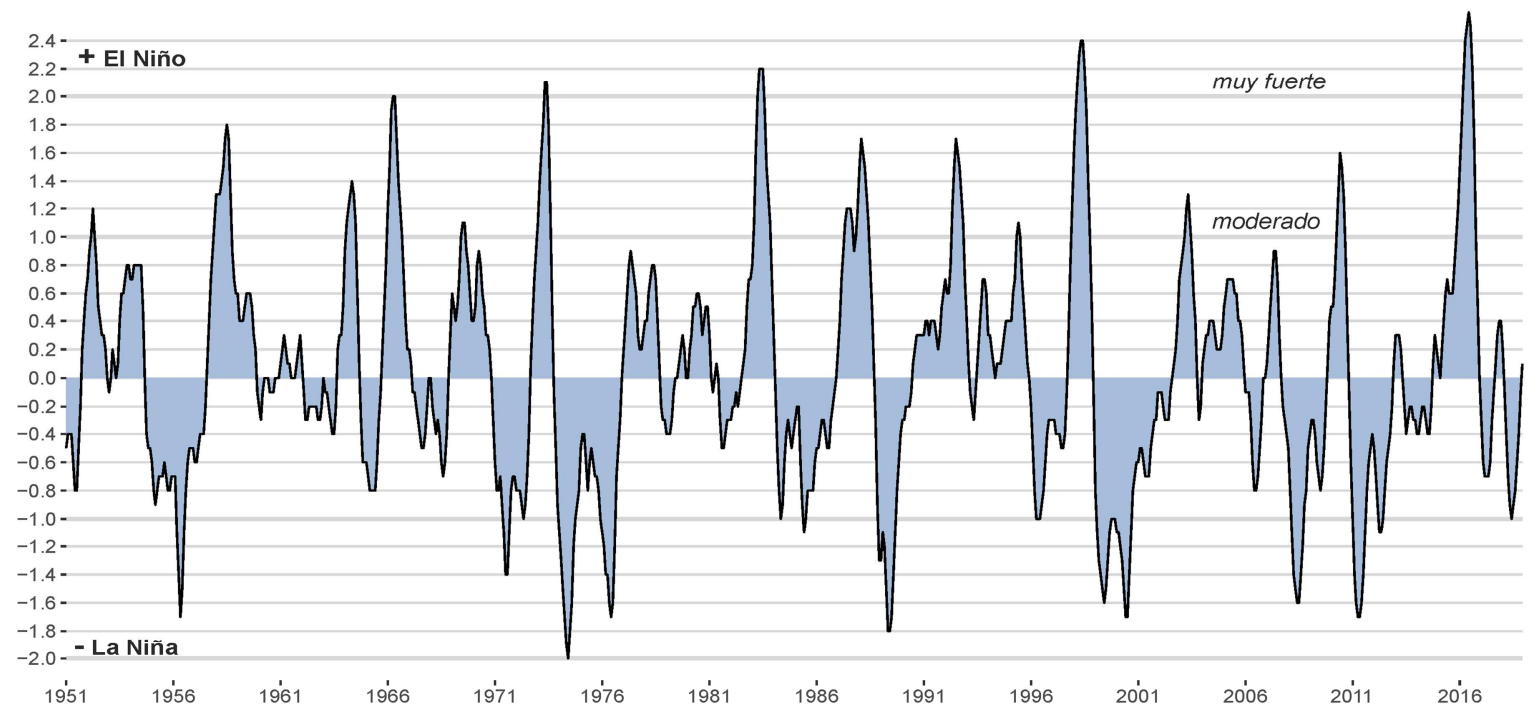

Figura 8. Evolución del índice ONI, anomalía de la temperatura superficial del mar durante tres meses, desde 1950 hasta 2017. Datos: http://origin.cpc.ncep.noaa.gov

La variabilidad intra- e interanual de la precipitación peninsular se ve influenciada no solo por los patrones de circulación del sector euroatlántico y la inestabilidad atmosférica, sino que también se encuentra afectada por el transporte de humedad desde el Atlántico norte y el Mediterráneo. Una de las principales fuentes de humedad, afectando a la precipitación en la PI corresponde a la región 
atlántica que se extiende desde el Golfo de México a las islas del Caribe (Gimeno et al. 2010, 2013, 2016). Otros estudios también han encontrado relaciones entre el fenómeno del Niño y las precipitaciones en Europa (Tabari and Willems, 2018; Lorenzo et al., 2010). El índice de Niño Oceánico (ONI) se ha convertido en el valor de referencia que la NOAA utiliza para identificar los eventos de El Niño y La Niña en el Pacífico tropical. Es la anomalía media de SST de 3 meses en ejecución para la región del Niño 3.4 (es decir, $5^{\circ} \mathrm{N}-5^{\circ} \mathrm{S}, 120^{\circ}-170 \mathrm{~W}$ ). Los eventos se definen como 5 períodos consecutivos de 3 meses que se superponen en/o por encima de la anomalía de +0.5 para eventos El Niño y en/o por debajo de la anomalía - 0.5 para eventos La Niña. Las tres sequias más severas sufridas en los últimos 11 años $(2005,2011-2012$ y 2017) en la PI coincidieron con eventos La Niña.

\section{Conclusiones}

En el análisis de la sequía de 2017 se observa que las anomalías de precipitación van parejas con las anomalías observadas en la fracción de nubosidad. Con repercusión en sectores tan dependientes de las condiciones meteorológicas como el sector agrícola o el de las energías renovables. Los patrones de circulación, tanto sinópticos como globales, son los que determinan las condiciones atmosféricas de una región concreta. En la sequía de 2017 se observa un comportamiento de la circulación atmosférica similar al registrado en las tres sequias más severas sufridas en los últimos 11 años (2005, 2011-2012 y 2017). NAO y EA/WR positivos y SCA negativo junto con eventos de La NIÑA en el Pacífico. Aunque los resultados de este estudio, usando particularmente la fracción de nubosidad, todavía son preliminares y precisarán de un mayor análisis, el hecho de que se repitan condiciones en los diferentes episodios de sequía nos podría abrir la posibilidad de llevar a cabo la elaboración de un sistema de alerta temprana que pudiera poner en aviso a los sectores más sensibles de la posibilidad de ocurrencia de un periodo de sequía severa.

\section{Referencias}

BAUM, B \& PLATNICK, S (2006). Introduction to MODIS cloud products. In J Qu, W Gao, M Kafatos, R Murphy, \& V Salomonson (Eds.), Earth Science Satellite Remote Sensing, pp. 74-91. Springer.

BAUM, B; MENZEL, W; Frey, R; Tobin, D; Holz, R; Ackerman, S; Heidinger, A; Yang, P (2012). MODIS cloud-top property refinements for collection 6. J Appl Meteor Climatol, 51:1145-1163.

CASANUEVA, A; RODRÍGUEZ-PUEBLA, C; FRÍAS, MD; GONZÁLEZ-REVIRIEGO, N (2014a). Variability of extreme precipitation over Europe and its relationships with teleconnection patterns, Hydrol Earth Syst Sci, 18:709-725.

DECASTRO, M; LORENZO, N; TABOADA, J; SARMIENTO, M; ALVAREZ, I; GOMEZ-GESTEIRA, $M$ (2006). Teleconnection patterns influence on precipitation variability and on river flow regimes in the Miño River basin (NW Iberian Peninsula). Climate Research, 32:63-73. 
DECASTRO, M; LORENZO, N; TABOADA, JJ; SARMIENTO, M; ALVAREZ, I; GOMEZ-GESTEIRA, M (2006). Influence of teleconnection patterns on precipitation variability and on river flow regimes in the Miño River basin (NW Iberian Peninsula). Clim Res, 32: 63-73.

ESTEBAN-PARRA, MJ; RODRIGO, FS; CASTRO-DÍEZ, Y (1998). Spatial and temporal patterns of precipitation in Spain for the period 1880-1992. Int J Climatol, 18:1557-1574.

FREY, R., ACKERMAN, S., LIU, Y., STRABALA, K, ZHANG, H, KEY, J, \& WANG, X (2008). Cloud detection with MODIS. part I: improvements in the MODIS cloud mask for collection 5. J. Atmos. Oceanic Technol., 25, 1057-1072.

GIMENO, L; DOMINGUEZ, F; NIETO, R; TRIGO, R; DRUMOND, A; REASON, CJC; TASCHETTO, AS; RAMOS, AM; KUMAR, R; MARENGO MAJOR, J (2016). Mechanisms of Atmospheric Moisture Transport and Their Role in Extreme Precipitation Events. Annual Review of Environment and Resources, 41:117-141.

GIMENO, L; DRUMOND, A; NIETO, R; TRIGO, R.M.; STOHL, A (2010). On the origin of continental precipitation. Geophys Res Lett, 37:L13804.

GIMENO, L; NIETO, R; DRUMOND, A; CASTILLO, R; TRIGO, R.M. (2013). Influence of the intensification of the major oceanic moisture sources on continental precipitation. Geophys Res Lett, 40:1-8.

HUTH, R; BECK, C; KUČEROVÁ, M (2016). Synoptic-climatological evaluation of the classifications of atmospheric circulation patterns over Europe. International Journal of Climatology, 36:27102726.

HUTH, R; BECK, C; PHILIPP, A; DEMUZERE, M; USTRNUL, Z; CAHYNOVÁ, M; KYSELÝ, J; TVEITO, OE (2008). Classifications of atmospheric circulation patterns: Recent advances and applications. Ann NY Acad Sci, 1147:105-152.

JENKINSON, AF \& COLLISON, FP (1977). An initial climatology of gales over the North Sea. Synoptic Climatology Branch Memorandum, 62, Meteorological Office, Bracknell.

JONES, PD; HULME, M; BRIFFA, KR (1993). A comparison of Lamb circulation types with an objective calssigication scheme. Int J Climatol, 13:655-663.

KINGSTON, DG; STAGGE, JH; TALLAKSEN, LM; Hannah, DM (2015). European-Scale Drought: Understanding Connections between Atmospheric Circulation and Meteorological Drought Indices. J. Climate, 28:505-516.

KOTARBA, A.Z. (2010). Estimation of fractional cloud cover for moderate resolution imaging spectroradiometer/terra cloud mask classes with high-resolution over ocean aster observations. Geophys Res, 115:D22210.

LORENZO, M.N.; TABOADA, J.J.; GIMENO, L (2008). Links between circulation weather types, teleconnection patterns, and their influence on precipitation patterns in Galicia (NW Spain). International Journal of Climatology, 28:1493-1505. 
LORENZO, M.N.; TABOADA, J.J.; IGLESIAS, I, GÓMEZ-GESTEIRA, M (2010). Predictability of the spring rainfall in Northwestern Iberian Peninsula from sea surfaces temperature of ENSO areas. Climatic Change, 107:329-341.

MUÑOZ-DIAZ, D \& RODRIGO, F (2006). Seasonal rainfall variations in Spain (1912-2000) and the links to atmospheric circulation. Atmos Res, 81:94-110.

PHILIPP, A (2009). Comparison of principal component and cluster analysis for classifying circulation pattern sequences for the European domain. Theor Appl Climatol, 96, 31-41.

PHILIPP, A; BARTHOLY, J; BECK, C; ERPICUM, M; ESTEBAN, P; FETTWEIS, X; HUTH, R; JAMES, P; JOURDAIN, S; KREIENKAMP, F; KRENNERT, T; LYKOUDIS, S; MICHALIDES, SC; PIANKO-KLUCZYNSKA, K; POST, P; ALVAREZ, DR; SCHIEMANN, R; SPEKAT, A; TYMVIOS, F (2010). Cost733cat - a database of weather and circulation type classifications. Phys Chem Earth (Special Issue), 35:360-373.

RAMOS, A.M.; BARRIOPEDRO, D.; DUTRA, E. (2015). Circulation weather types as a tool in atmospheric, climate, and environmental research. Frontiers in Environmental Science, 3.

RODRÍGUEZ-PUEBLA, C.; ENCINAS, A.H.; NIETO, S.; GARMENDIA, J. (1998). Spatial and temporal patterns of annual precipitation variability over the Iberian Peninsula. Int J Climatol, 18:299-316.

RODRÍGUEZ-PUEBLA, C.; ENCINAS, A.H.M.; Sáenz, J. (2001). Winter precipitation over the Iberian Peninsula and its relationship to circulation indices. Hydrol Earth Syst Sci, 5:233-244.

ROYÉ, R.; LORENZO, N.; RASILLA, D.; MARTÍ, A. (2018). Spatio-temporal variations of cloud fraction based on circulation types in the Iberian Peninsula. International Journal of Climatology.

TABARI, H. \& WILLEMS, P. (2018). Lagged influence of Atlantic and Pacific climate patterns on European extreme precipitation. Scientific Reports, 8:5748.

TABARI, H. \& WILLEMS, P. (2018). More prolonged droughts by the end of the century in the Middle East. Environ Res Lett, 13.

TOLLER, G.; XIONG, X.; SUN, J.; WENNY, B.; GENG, X.; KUYPER, J.; ANGAL, A.; CHEN, H.; MADHAVAN, S.; WU, A. (2013). Terra and Aqua Moderate-resolution Imaging Spectroradiometer collection 6 level 1b algorithm. J Appl Remote Sens, 7:073557.

TRIGO, I. (2006). Climatology and interannual variability of storm tracks in the euro-atlantic sector: a comparison between era-40 and ncep/ncar reanalyses. Clim Dyn, 26:127-143.

TRIGO, R. \& DA CAMARA, C. (2000). Circulation weather types and their influence on the precipitation regime in Portugal. Int J Climatol, 20:1559-1581.

TRIGO, R.M.; VALENTE, M.A.; TRIGO, I.F.; MIRANDA, P.M.A.; RAMOS, A.M.; PAREDES, D.; GARCíA-HERRERA, R. (2008). The impact of North Atlantic wind and cyclone trends on European precipitation and significant wave height in the Atlantic. Ann NY Acad Sci (Spec Issue Trends Dir Clim Res), 1146:212-234.

VERMOTE, E., \& WOLFE, R. (2015). MOD09GA/MYD09GQ MODIS/Terra Surface Reflectance Daily L2G Global 1kmand 500m SIN Grid V006 [Dataset]. NASA EOSDIS LP DAAC.. doi:10.5067/MODIS/MYD09GQ.006. 